\title{
Corrigendum
}

\section{Effect of phase feeding and valine-to-lysine ratio during lactation on sow and piglet performance ${ }^{1}$}

\author{
A. Craig, ${ }^{*, \dagger, 2}$ W. Henry, ${ }^{\ddagger}$ and E. Magowan ${ }^{\dagger}$ \\ *Agri-Food and Bioscience Institute, Hillsborough, Northern Ireland, BT26 6DR; 'Q Queens University Belfast, \\ Belfast, Northern Ireland, BT1 7NN; and Rekify Ltd, Co. Armagh, Northern, Ireland
}

(C) The Author(s) 2019. Published by Oxford University Press on behalf of the American Society of Animal Science. All rights reserved. For permissions, please e-mail: journals.permissions@oup.com.

J. Anim. Sci. 2019.97:981

doi: 10.1093/jas/sky444

In "Effect of phase feeding and valine-to-lysine ratio during lactation on sow and piglet performance" by A. Craig et al [Journal of Animal Pages 3835-3843, https://doi.org/10.2527/ jas.2016-0648], values for SID in Table 2 had been calculated on the formulated instead of actual. Science, Volume 94, Issue 9, 1 September 2016, The updated table now contains the correct values.

Table 2: Formulated and actual analysis of experimental diets on an 'as fed' (wet) basis

\begin{tabular}{|c|c|c|c|c|c|}
\hline & \multirow[b]{2}{*}{ Control } & \multicolumn{2}{|c|}{ Flat } & \multicolumn{2}{|c|}{ Phased } \\
\hline & & Low Valine & Norm Valine & Low Valine & Norm Valine \\
\hline \multicolumn{6}{|l|}{ Formulated Composition } \\
\hline $\mathrm{DE}^{1}, \mathrm{MJ} / \mathrm{kg}$ & 13.5 & 14.4 & 14.4 & 15.0 & 15.0 \\
\hline $\mathrm{CP}, \mathrm{g} / \mathrm{kg}$ & 170 & 198 & 198 & 199 & 200 \\
\hline Crude Fiber, g/kg & 359 & 266 & 259 & 250 & 251 \\
\hline $\mathrm{NDF}, \mathrm{g} / \mathrm{kg}$ & 142 & 112 & 109 & 106 & 106 \\
\hline Lysine, $g / k g$ & 10.0 & 12.5 & 12.5 & 14.0 & 14.0 \\
\hline Valine, $\mathrm{g} / \mathrm{kg}$ & 8.2 & 9.5 & 15.0 & 11.0 & 16.8 \\
\hline Valine:Lysine & $0.82: 1$ & $0.76: 1$ & $1.2: 1$ & $0.79: 1$ & $1.2: 1$ \\
\hline \multicolumn{6}{|l|}{ Actual composition } \\
\hline $\mathrm{DE}^{1}, \mathrm{MJ} / \mathrm{kg}$ & 13.6 & 14.1 & 14.4 & 14.9 & 15.1 \\
\hline $\mathrm{CP}, \mathrm{g} / \mathrm{kg}$ & 144 & 159 & 174 & 156 & 162 \\
\hline $\mathrm{NDF}, \mathrm{g} / \mathrm{kg}$ & 106 & 91 & 91 & 79 & 91 \\
\hline Lysine, $\mathrm{g} / \mathrm{kg}$ & 8.8 & 9.2 & 10.0 & 11.1 & 9.6 \\
\hline Valine, $\mathrm{g} / \mathrm{kg}$ & 6.7 & 6.2 & 12.0 & 7.7 & 10.3 \\
\hline Valine:Lysine & $0.76: 1$ & $0.67: 1$ & $1.2: 1$ & $0.69: 1$ & $1.07: 1$ \\
\hline SID ${ }^{2}$ Lysine, $\mathrm{g} / \mathrm{kg}$ & 7.2 & 7.5 & 8.2 & 9.1 & 7.9 \\
\hline SID $^{2}$ Methionine, $\mathrm{g} / \mathrm{kg}$ & 2.2 & 2.3 & 2.5 & 2.7 & 2.4 \\
\hline $\mathrm{SID}^{2} \mathrm{M}+\mathrm{C}, \mathrm{g} / \mathrm{kg}$ & 4.2 & 4.4 & 4.8 & 5.4 & 4.7 \\
\hline SID $^{2}$ Tryptophan, g/kg & 1.4 & 1.4 & 1.6 & 1.7 & 1.5 \\
\hline SID $^{2}$ Threonine, $\mathrm{g} / \mathrm{kg}$ & 4.7 & 4.9 & 5.3 & 5.9 & 5.1 \\
\hline SID $^{2}$ Valine, $\mathrm{g} / \mathrm{kg}$ & 5 & 5.3 & 5.7 & 6.4 & 5.5 \\
\hline
\end{tabular}

